functionality, gender, physical exercise, hemodialysis complications, marital status, educational level and anxiety levels. Women exhibited a statistically significant higher state anxiety levels compared to men. Widowed participants, that is, people living alone who had experienced the loss of a beloved one, reported higher state, trait and total anxiety levels. Finally, people with higher education showed lower trait and total anxiety. Individuals who were capable of taking care of themselves reported lower state, trait and total anxiety levels with a statistically significant difference compared to those who needed external help. Additionally, those who physically exercised exhibited statistically significant lower state, trait and total anxiety levels compared to those who didn't exercise.

Conclusions: The conclusions of the present study could be a critical point in determining guidelines that would substantially contribute to the improvement of the psychological state of patients undergoing hemodialysis treatment.

\title{
528 - Health locus of control beliefs among older Greek hemodialysis patients: Sociodemographic and psychological correlates
}

Afroditi Zartaloudi ${ }^{1}$, Sofia Kalini ${ }^{2}$, Anna Kavga ${ }^{1}$, Aggeliki Stamou ${ }^{1}$

${ }^{1}$ University of West Attica, Athens, Greece

${ }^{2}$ Graduate of the post-graduate program "Neurological Diseases: Evidence based practice", University of West Attica, Athens, Greece

\begin{abstract}
Introduction: Quality of life of a significant proportion of patents under hemodialysis treatment is determined by their health locus of control beliefs (internal, external, chance).

The aim of the present study was to investigate health locus of control of patients under hemodialysis treatment. Moreover, the relationship between socio-demographic, clinical, psychological factors and health locus of control was explored.
\end{abstract}

Method: A total of 105 patients with chronic kidney failure under hemodialysis treatment (64 men and 41 women) with a mean age of $72.51 \pm 7.55$ years were recruited and completed (a) a demographic questionnaire, (b) the Multidisciplinary Health Locus of Control Scale and (c) the State-Trait Anxiety Inventory.

Results: Internal locus of control (the degree to which participants feel they are in control of their own health) fluctuates lower, while locus of control from others (the degree that participants believe that others -doctors- control their health) and luck (the degree to which participants believe their health is a matter of luck) fluctuates higher in our participants than general population. State, trait and total anxiety levels were significantly correlated negatively with internal locus of control and positively with "chance" locus of control scale in the present study.

Conclusions: Our participants seemed to feel highly dependent from others, such as treating physicians, nurses, family, hemodialysis machine resulting in a limited sense of freedom and autonomy. Understanding health locus of control beliefs among Greek hemodialysis patients may help health professionals to implement appropriate counseling intervention programs and improve patient psychological state and provided services. 